\title{
MICROSYSTEM FOR PARTICLE COUNTING AND SIZING WITH TUNABLE SENSITIVITY AND THROUGHPUT
}

\author{
T.E. Winkler ${ }^{l}$, H. Ben-Yoav ${ }^{l}$, D.L. Kelly ${ }^{2}$, and R. Ghodssi $i^{*}$ \\ ${ }^{1}$ University of Maryland, College Park, Maryland, USA \\ ${ }^{2}$ University of Maryland, Baltimore, Maryland, USA
}

\begin{abstract}
We present the first comprehensive study of integrating hydrodynamic focusing to provide tunable sensitivity and throughput in impedance cytometry. This interplay has never been investigated with respect to particle counting and sizing performance. Our microfluidic lab-on-a-chip (LOC) differs from previous designs in the added tunability while featuring simpler fabrication. Here, we demonstrate a 2.7 -fold increase in sensitivity with hydrodynamic focusing. Systematic studies of the coupling between integrated LOC components enable optimal utilization of microsystem capabilities.
\end{abstract}

\section{INTRODUCTION}

Characterization of large quantities of individual particles is highly relevant to multiple fields, particularly blood analysis [1]. A simple cell count is useful, e.g. to diagnose anemia, but the utility of such measurements increases significantly with the ability to also determine size, surface markers, and interior composition. Applications range from CD4 T-cell monitoring in cases of HIV to stem cell characterization in research. The current gold standard for such measurements is bulky benchtop flow cytometers. These rely on a focused stream of blood cells being subjected to multiple analysis methods, involving fluorescent labels for populationspecific surface antigens (e.g. CD4, CD8, ...), laser light scattering, and absorbance, or impedance measurements. Flow cytometers allow for highly accurate analysis, but rely on labels and complex optics. These factors are major barriers in bringing this technology to the point of care (POC), where it would benefit patients as well as physicians by providing immediate results and increasing accessibility, especially in remote locations [2].

The impedance measurement aspect, however, is ideally suited for integration into small, portable lab-on-a-chip (LOC)-type devices, with electronics having a long history of miniaturization. The approach in its most basic form applies the Coulter principle [1]. As a particle (or cell) of diameter $d_{p}$ passes through an aperture of diameter $D_{A}$ between two chambers, it causes a change in impedance $\Delta Z$ measured between two electrodes on either side of the aperture. A first-order approximation for this change is $\Delta Z=\frac{4 \rho_{m} d_{p}^{3}}{\pi D_{A}^{4}}$, where $\rho_{m}$ is the resistivity of the electrolyte. Consequently, this signal can be used to differentiate particles based on their size. By extension into the alternating current domain, this allows for probing more generalized changes in dielectric properties caused by particles within the interaction volume [3].

Multi-frequency impedance cytometry was presented as an attractive method for multi-dimensional single-cell analysis in LOC systems [4], [5]. However, current implementations still suffer from limited resolution, and employ multi-layer fabrication processes. Here, we propose an LOC combining impedance cytometry with hydrodynamic focusing for adjustable throughput (sample volume/time) and improved performance. This relies on constricting the sample flow through a virtual aperture $(V A)$ of a non-conductive fluid. In contrast to physical channel confinement, this is inherently more versatile and limits the danger of channel clogging. While flow focusing has been utilized to enhance the performance of coulter counter-type devices, to date no systematic study has been conducted on the interplay between flow ratios, particle sizing sensitivity, and throughput [6]. It is only through such studies, both in models and experiments, that optimal utilization of microsystem capabilities becomes possible.

\section{MATERIALS AND METHODS Design}

We propose a simple design, shown in Figure 1, comprising two physical layers. The first layer incorporates four pairs of microelectrodes for impedance measurements $(25 \mu \mathrm{m}$ wide, $25 \mu \mathrm{m}$ gap). The microfluidic channels constitute the second layer, with a cross-section of $75 \times 20 \mu \mathrm{m}^{2}$ (width $\times$ height).

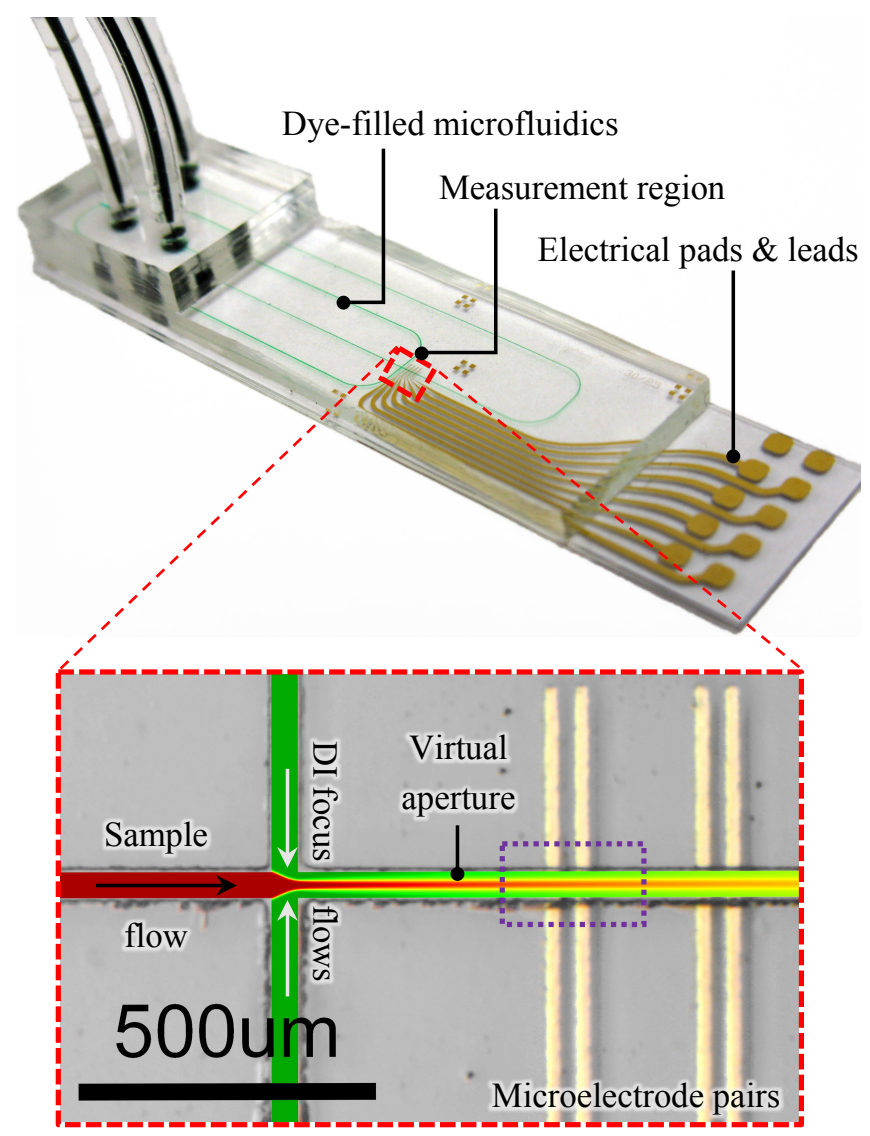

Figure 1: Top: Photograph of our impedance cytometry LOC. The microfluidic channels were filled with dye to enhance visualization. Bottom: Micrograph of the measurement region highlighted by the red dashed box in the photograph. Hydrodynamic focusing is schematically illustrated by overlaid FEM simulation for a ratio of sample (particles in electrolyte; red) to $\mathrm{DI}-\mathrm{H}_{2} \mathrm{O}$ focus (green) flows of $\mathrm{FR}=1: 1$. The virtual aperture effect is conserved downstream, where impedance is measured across the gold microelectrode pairs. The purple dotted box indicates the region simulated by electrodynamic FEM (Figure 2). 
We made extensive use of finite element modeling (FEM), in combination with equivalent circuit modeling, to guide the design process, as described below. Critical parameters such as channel cross-section and electrode gap were chosen based on model optimization.

\section{Fabrication}

Gold (200 nm; on $20 \mathrm{~nm}$ chrome adhesion layer) was deposited on a pyrex wafer by sputtering. We transferred the electrode pattern through contact photolithography in Shipley 1813 positive photoresist, followed by wet etching. The wafer was diced, and the chips were cleaned by immersion piranha solution (sulfuric acid and hydrogen peroxide, 3:1 ratio) to remove any organic contaminants.

For the microfluidic layer, a mold was created using SU-8 2015 negative photoresist patterned on silicon using contact photolithography. Using this master, we cast channels from poly(dimethylsiloxane) (PDMS). After thermal curing at $60^{\circ} \mathrm{C}$, the PDMS was diced and $2 \mathrm{~mm}$ diameter fluidic connections were punched.

We utilized contact profilometry and optical microscopy to confirm that all dimensions were within $10 \%$ of the design parameters. A combination of oxygen plasma and $80{ }^{\circ} \mathrm{C}$ thermal treatment was applied to irreversibly bond both layers for individual devices.

\section{Modeling}

Finite element modeling (FEM) was performed in COMSOL Multiphysics (COMSOL, Inc.; Palo Alto, CA), using the MEMS and Microfluidics packages.

The 2D hydrodynamic model considered a slow-diffusing species (particles) and a fast-diffusing species (ions) introduced through a center sample channel, focused symmetrically by deionized water $\left(\mathrm{DI}-\mathrm{H}_{2} \mathrm{O}\right)$ flows. A representative simulation is overlaid on the micrograph in Figure 1 (inset). The main model outputs of interest are the cross-sectional concentration profiles $125 \mu \mathrm{m}$ downstream from the flow focusing inlets - the respective full width at half maximum $(F W H M)$ for the ions can be considered equivalent to the $V A$ width.

The 3D electrodynamic model simulates a particle of radius $r$, conductivity $\sigma$, and permittivity $\varepsilon$, suspended in a section of microfluidic channel between two coplanar electrodes. To approximate the impact of hydrodynamic focusing, we incorporate two distinct environments (apart from the particle) within the channel segment - electrolyte in the center at a certain width corresponding to $V A$, DI- $\mathrm{H}_{2} \mathrm{O}$ towards the sides. A sample model geometry is displayed in Figure 2. The model output is the change in impedance $|\Delta Z|$ measured across electrodes between particle and no-particle conditions.

\section{Experiments}

The fabricated LOCs were connected to syringes using Tygon tubing (Cole-Parmer; Vernon Hills, IL). Constant flow was provided through syringe pumps (KDS230 (KD Scientific, Inc.; Holliston, MA), Genie Plus (Kent Scientific Corporation; Torrington, CT), NE-300 (New Era Pump Systems, Inc.; Farmingdale, NY)). Admittance measurements for model verification were done using a VSP-300 potentiostat (Bio-Logic; Claix, France). Impedance cytometry data was recorded via LabView utilizing an E4980A Precision LCR Meter (Agilent; Santa Clara, CA). The background signal was determined through MATLAB (MathWorks, Inc.; Natick, MA) robust local regression smoothing of the raw data, the signal peaks using a peak finding algorithm. Population averages were calculated using histogram

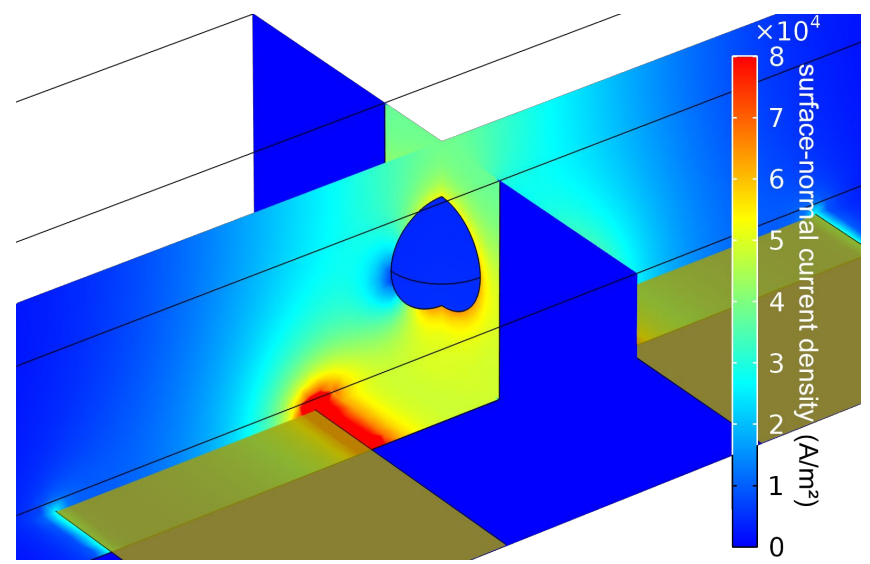

Figure 2: Exemplary electrodynamic COMSOL simulation result with particle (center; blue sphere) suspended in channel between two electrodes (bottom; gold rectangles). This matches the region highlighted by the purple dotted box in the inset to Figure 1. The colors correspond to the surface-normal current density from blue (low) to red (high), clearly illustrating the particle (no conduction), the focused electrolyte in the center of the channel (green, good conduction), and the virtual aperture of pure water on either side (blue, no conduction).

peak fits in OriginPro (OriginLab Corporation; Northampton, MA).

Prior to use, the LOCs were rinsed with Fetal Bovine Serum (FBS; Life Technologies; Carlsbad, CA) to reduce PDMS hydrophobicity. Polystyrene particles $(r=3 \mu \mathrm{m}$ and $5 \mu \mathrm{m}$; sulfatetype) were purchased from Life Technologies (Carlsbad, CA) and suspended in phosphate-buffered saline (PBS; 1x from tablet; Sigma-Aldrich; St. Louis, MO). To reduce settling velocity through density matching, sucrose (Sigma-Aldrich; St. Louis, MO) was added to $14 \% \mathrm{w} / \mathrm{v}$. All solutions were based on $\mathrm{DI}-\mathrm{H}_{2} \mathrm{O}$ $(\rho=18 \Omega \mathrm{cm})$.

\section{RESULTS AND DISCUSSION}

\section{Hydrodynamic Model and Validation}

The flow rates are the main external parameters to control the $V A$ width, the critical parameter for impedance cytometry performance. To elucidate their correlation, we utilize hydrodynamic FEM to determine the ionic $F W H M$ for a range of flow ratios $(F R)$ of $\mathrm{PBS}$-based sample to $\mathrm{DI}-\mathrm{H}_{2} \mathrm{O}$ focus. The

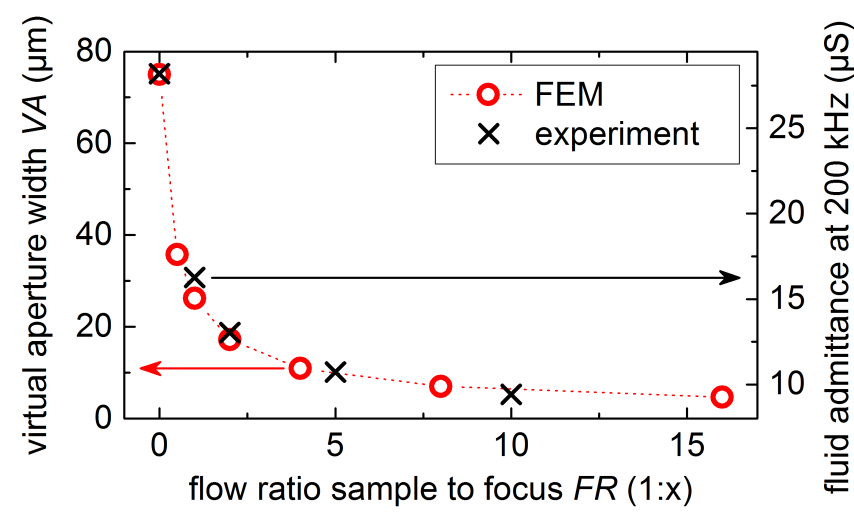

Figure 3: FEM simulation showing the dependence of VA width on FR (red circles). Experimental verification through fluid admittance measurements in the LOC (black crosses). 
results, plotted in Figure 3, are in qualitative agreement with theory [7]. As expected intuitively, the virtual aperture width decreases drastically as flow focusing is introduced, with the effect saturating at high flow ratios. The behavior is independent of flow rate, at least in the laminar flow regime.

To verify these results in our LOC, we introduced a pure PBS sample flow $(10,20,50,100 \mu \mathrm{l} / \mathrm{h})$ and DI-H2O focus flows ( $100 \mu \mathrm{l} / \mathrm{h}$ combined). To achieve $\mathrm{FR}=0$, PBS was substituted for the DI-H2O. We measured the admittance of the fluid across electrodes, which at $200 \mathrm{kHz}$ is dominated by ionic conduction. Thus, this parameter is expected to linearly correlate with the ionic FWHM. Indeed, the overlaid experimental data in Figure 3 aligns well with model results. The fact that the measured trend is toward a non-zero value at high FR can be attributed to parasitic currents in the real-world instrument-LOC circuit.

\section{Electrodynamic Model}

To illustrate the advantages of hydrodynamic focusing in impedance cytometry, we initially rely on electrodynamic FEM. In Figure 4, we display the relative $|\Delta Z|$ (i.e., as a percentage of the empty-channel $Z$ ) induced by an $r=5 \mu \mathrm{m}$ particle for a range of $V A$ widths. We found a frequency of $f=200 \mathrm{kHz}$ to be most sensitive to resistive properties, and thus $r$, and this was used throughout this work. The plot shows data for channel widths of $25 \mu \mathrm{m}$ and $50 \mu \mathrm{m}$, revealing the signal is independent of the actual channel width (memory constraints prevented simulations for $75 \mu \mathrm{m}$ width). Therefore, at the chosen $f$, the $V A$ is expected to function identical to a physical constriction. As expected, the relative $|\Delta Z|$ induced by an $r=5 \mu \mathrm{m}$ particle increases significantly with decreasing $V A$. Specifically, reducing the aperture from $50 \mu \mathrm{m}$ to $5 \mu \mathrm{m}$ enhances the signal 10 -fold from $|\Delta Z|=1.8 \%$ to $|\Delta Z|=18 \%$. At very low $V A \leq r$, the signal saturates, which can be attributed to the fact that in this regime, only part of the particle is in electrolyte and thus contributing to the signal. We expect that in reality, a boundary layer of PBS would surround the particle, which would alter the results. However, considering the underlying approximation of a well-defined boundary between PBS and DI- $\mathrm{H}_{2} \mathrm{O}$ in this model, the additional error introduced by the omitted particle boundary layer is likely negligible.

\section{Integrated Lab-on-a-Chip}

In Figure 5, we illustrate the impedance-based particle counting principle using our LOC with a mixture of both $r=3 \mu \mathrm{m}$ and $5 \mu \mathrm{m}$ bead populations at a sample flow of $45 \mu \mathrm{l} / \mathrm{h}, F R=1: 0$.

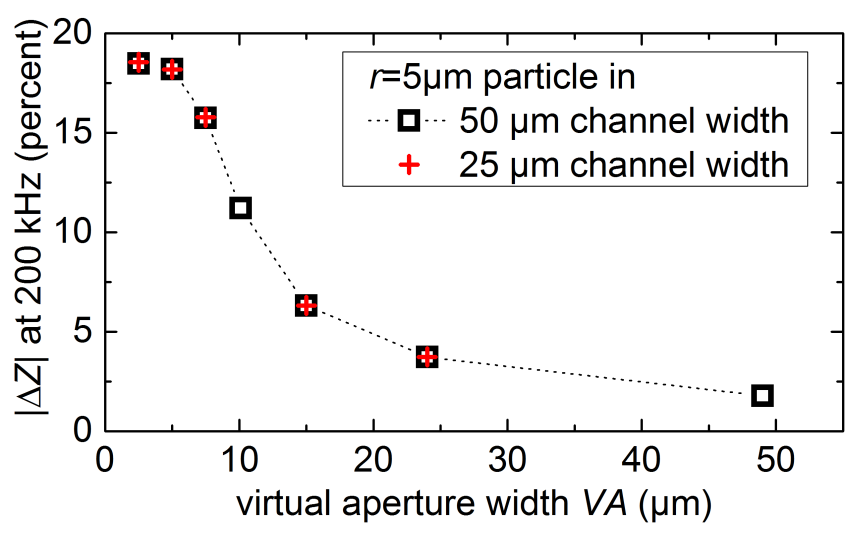

Figure 4: FEM simulations predict flow focusing can significantly enhance impedance-based particle measurements. The effect is independent of actual channel width (black squares, red plusses).

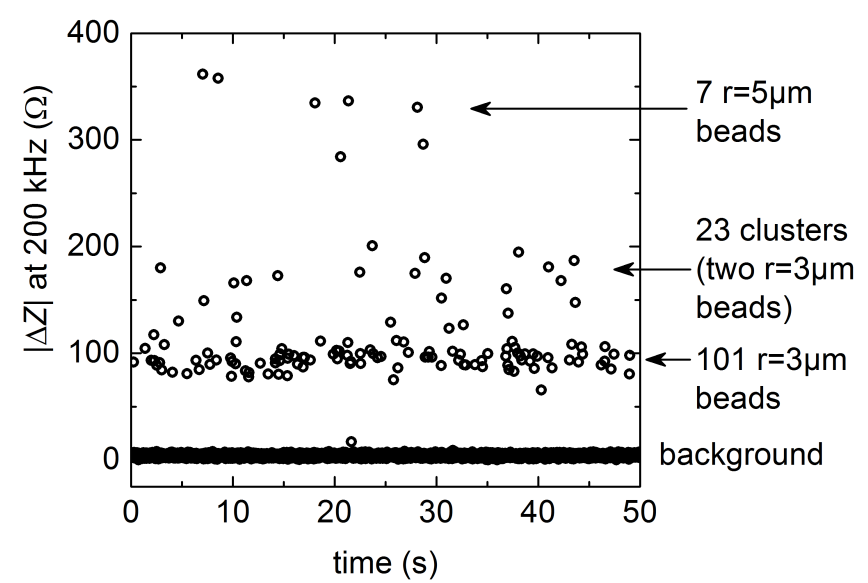

Figure 5: Exemplary experimental $|\Delta Z|$ data recorded using LOC with mixture of $r=3 \mu \mathrm{m}$ and $5 \mu \mathrm{m}$ beads at $F R=1: 0$.

The MATLAB-processed data shows distinct peaks in $|\Delta Z|$ corresponding to particles passing between the electrodes. Furthermore, three distinct populations become apparent, corresponding in order of increasing signal to $3 \mu \mathrm{m}$ beads $(|\Delta Z|=94 \pm 9 \Omega)$, clusters of two $3 \mu \mathrm{m}$ beads $(|\Delta Z|=162 \pm 23 \Omega)$, and $5 \mu \mathrm{m}$ beads $(|\Delta Z|=329 \pm 29 \Omega)$. While clusters of three or four $3 \mu \mathrm{m}$ beads are statistically unlikely, their mis-identification as a $5 \mu \mathrm{m}$ bead cannot be ruled out in this data due to their similar volume. Although the $3 \mu \mathrm{m}$ bead population signal is welldefined, we note a larger spread in the cluster signals, which is in line with their non-spherical shape - based on orientation relative to the electrodes, the signal magnitude is expected to vary, as the electric field is not isotropic. In future work with particle mixtures, this may enable more definitive differentiation between clusters of small particles and larger single particles through analysis of the transient signal during passage between the electrodes.

To determine the impact of hydrodynamic focusing on sensitivity of our device, we utilized single-population samples of beads, and varied $F R$ while keeping the total flow rate (sample + focus) constant at $45 \mu \mathrm{l} / \mathrm{h}$. From histograms based on data

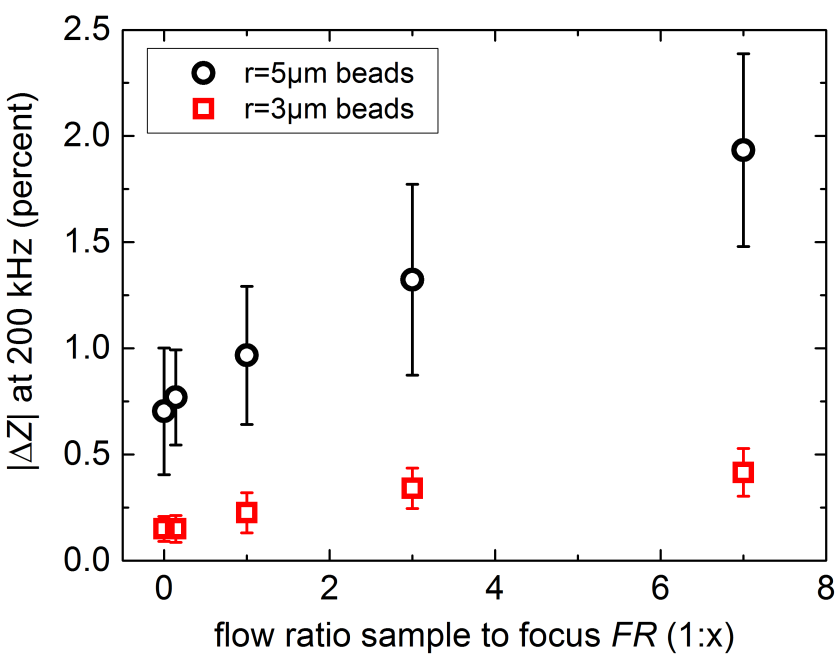

Figure 6: Experimental $|\Delta Z|$ recorded using LOC for both $r=3 \mu \mathrm{m}$ and $5 \mu \mathrm{m}$ beads increases significantly with increased $F R$, as does the relative separation between those populations. 
analogous to that shown in Figure 5, we produce Figure 6, plotting the average $|\Delta Z|$ for separate bead populations as a function of $F R$. The graph indicates up to $276 \%$ enhanced size-based differentiation, from $\Delta|\Delta Z|=0.55 \%$ to $\Delta|\Delta Z|=1.52$. Underlying this are overall increases in $|\Delta Z|$ by $277 \%$ and $275 \%$ for $3 \mu \mathrm{m}$ and $5 \mu \mathrm{m}$ beads, respectively. These numbers highlight the tunable sensitivity enabled through our approach.

While the trend agrees with modeling (Figure 4), the magnitudes are lower than predicted. Specifically, at $F R=1: 7$, modeling predicts $V A \approx 8 \mu \mathrm{m}$ (Figure 3 ), and in consequence $|\Delta Z| \approx 15 \%$ for $r=5 \mu \mathrm{m}$ beads (Figure 4). This almost order-ofmagnitude difference compared to experimental results warrants further investigation. One potential explanation is the aforementioned model assumption of well-defined boundaries between PBS and DI- $\mathrm{H}_{2} \mathrm{O}$ in the electrodynamic FEM. However, this is unlikely to be solely responsible for the discrepancy. Experimental causes such as parasitic capacitances, which become more dominant at high absolute $Z$ (correlating with higher $F R$ ), will need to be explored.

Overall, separation efficiency increases with $F R$; at the same time, the sample throughput (equaling sample input flow rate) in our experiments decreases (as total flow is kept constant). However, the sample flow rate is inherently independent from $F R$. In the laminar flow regime, sensitivity and throughput are thus decoupled in our LOC, enabling tailoring of these parameters to the specific experimental needs.

\section{CONCLUSIONS}

In conclusion, our LOC provides a simple yet efficient platform for impedance cytometry. We utilize it to systematically study the integration of hydrodynamic focusing. Thereby, we demonstrate a 2.7-fold improvement in signal amplitude, as well as a size-based population separation enhancement of similar magnitude. Compared to physical channel constriction, this approach offers a distinctly lower risk of clogging, and provides tunable, rather than static, sensitivity and throughput. Extensive modeling provides the underpinning for our design and corroborates experimental results such as the relationship between flow ratios and virtual apertures. It also provides guidance for future investigations, as simulations predict even higher performance increases due to flow focusing.

Our design allows for straightforward expansion to multifrequency signal recording. This will provide high-resolution multi-dimensional particle characterization towards an integrated microsystem capable of full differential blood cell counting. Furthermore, our approach illustrates the benefits of model-guided design as well as in-depth study of systems interplay in LOC devices. With many examples of exciting LOC technologies sensors, blood handling components, microfluidic components published in the literature, we believe it is only through such studies that they can be optimally realized in integrated POC microsystems.

\section{ACKNOWLEDGEMENTS}

The authors would like to thank the Robert W. Deutsch Foundation and the Maryland Innovation Initiative for financial support. The authors likewise appreciate the support of the Maryland NanoCenter and its FabLab. Travel support has been generously provided by the Transducer Research Foundation.

\section{REFERENCES}

[1] K. Kottke-Marchant and B. Davis, Laboratory Hematology Practice, John Wiley \& Sons, Ltd, Chichester, 2012.

[2] H. Craighead, "Future lab-on-a-chip technologies for interrogating individual molecules", Nature, 442, 387-393 (2006).

[3] T. Sun and H. Morgan, "Single-cell microfluidic impedance cytometry: a review", Microfluidics and Nanofluidics, 8, 423-443 (2010).

[4] D. Holmes, D. Pettigrew, C.H. Reccius, J.D. Gwyer, C. van Berkel, J. Holloway, D.E. Davies, and H. Morgan, "Leukocyte analysis and differentiation using high speed microfluidic single cell impedance cytometry", Lab on a Chip, 9, 2881 (2009).

[5] X. Han, C. van Berkel, J. Gwyer, L. Capretto, and H. Morgan, "Microfluidic Lysis of Human Blood for Leukocyte Analysis Using Single Cell Impedance Cytometry", Analytical Chemistry, 84, 1070-1075 (2012).

[6] X. Xuan, J. Zhu, and C. Church, "Particle focusing in microfluidic devices", Microfluidics and Nanofluidics, 9, 116 (2010).

[7] G.-B. Lee, C.-C. Chang, S.-B. Huang, and R.-J. Yang, "The hydrodynamic focusing effect inside rectangular microchannels", Journal of Micromechanics and Microengineering, 16, 1024 (2006).

\section{CONTACT}

*R. Ghodssi, tel: +1-301-405-8158; ghodssi@umd.edu 